\title{
Physician's dilemma in treating Acinetobacter baumanii: A combination of art and science
}

\author{
S. Khadanga ${ }^{1}$, T. Karuna ${ }^{2}$, P.K. Thatoi ${ }^{3}$, R. Moanty ${ }^{4}$, N. Mohapatra ${ }^{5}$ \\ ${ }^{1}$ Assistant Professor, Department of General Medicine, AlIMS Bhopal, Madhya Pradesh, India, ${ }^{2}$ Assistant Professor, Department of \\ Microbiology, AlIMS Bhopal, Madhya Pradesh, India, ${ }^{3}$ Assistant Professor, Department of Medicine, SCB Medical College, Odisha, India, \\ ${ }^{4}$ Professor, Department of Medicine, SCB Medical College, Odisha, India, ${ }^{5}$ Associate Professor, Department of Medicine, SCB Medical \\ College, Odisha, India
}

\section{A B S T R A C T}

\begin{abstract}
Acinetobacter species are gram negative coccobacilli and ubiquitous in nature. ${ }^{1}$ Quite frequently they are recovered from inpatients who are hospitalized for prolonged illnesses and often multiple times. Although originally considered to be a low grade opportunistic pathogen Acinetobacter species has emerged to be one of the common nosocomial pathogen, these isolates are often Multi-Drug Resistant (MDR). ${ }^{1}$ It still remains a physician's dilemma whether to treat such isolates or not, considering them pathologens or commmensals. Among the two Acinetobacter isolate, one was isolated as a commensal and the patient did not require any antibiotics. On the other hand, the second case succumbed just after we started the susceptible antibiotic.
\end{abstract}

Key words: Acinetobacter baumanii, Commensal, MDR (multi drug resistant), Nosocomial

\section{INTRODUCTION}

Though Acinetobacter are highly prevalent in the environment, they are only occasionally been cultured from the moist skin of healthy human beings. ${ }^{2}$ They have been commonly isolated from the hospitalized inpatients especially in ICU settings in patients who are immunocompromised and/or having co morbid conditions. ${ }^{1}$ The mechanical respiratory tract and IV devices are the most common sources of bacterimia. Infections of the colonized urinary tract, postoperative sites, biliary stents, Para nasal sinuses, eye and peritoneum are less common. ${ }^{1}$

Among many Acinetobacter species; A. baumanii is the most commonly isolated species and A. lwoffi come second in the list. ${ }^{1}$ Until recently $A$. baumanii was best known as a causative agent of nosocomial infection. However it has been described to cause severe acquired pneumonia and meningitis. ${ }^{1}$ Acinetobacter species are often extensively resistant to commonly available antimicrobial agents like that of antipseudomonal penicillins, $3^{\text {rd }}$ generation Cephalosporins, Fluoroquinolones, Aminoglycosides and Aztreonam. ${ }^{2}$ Polymyxin-B or colistin has been used with some success and carbapenams (Imipenam and meropenam) are often active against these species. ${ }^{3}$ Empirical combination therapy has been advocated when A. baumanii is isolated, pending susceptibility test. ${ }^{3,4}$

\section{CASE REPORT-1}

A 72 year old known diabetic and hypertensive patient presented to the emergency department for incoherent talk since last two days. The patient was on sustained release Metformin $1 \mathrm{Gm}$, Glemiperide $2 \mathrm{mg}$, Losartan Hydrochlorothiazide $(50 \mathrm{mg}+12.5 \mathrm{mg})$. Twelve days prior to this event the patient was hospitalized in this institution for severe falciparum malaria (cerebral + hepatopathy). He was treated with Artesunate, ceftriaxone and was put on indwelling catheter to prevent bed wetting.

At the time of admission, the patient was afebrile, pulse $90 / \mathrm{min}, \mathrm{BP}-140 / 90 \mathrm{~mm} \mathrm{Hg}$, respiratory rate was $16 /$ min. Chest, heart and abdominal examination was not noteworthy. There was no focal neurological deficit. The patient was admitted with a provisional diagnosis 
of metabolic encephalopathy with differential diagnosis of partially treated malaria, urosepsis or lacunar vascular stroke. Routine hematological and biochemical parameters were within normal limits except the serum Sodium was $115 \mathrm{meqv} / \mathrm{lt} \&$ Potassium was $4.3 \mathrm{meqv} / \mathrm{lt}$. ECG, X-ray chest and CT Scan of the brain were unremarkable. Blood and urine cultures reports were awaited.

The patient was given oral supplementation of common salt \& the patient recovered his sensorium gradually over 48-60 hrs. On the $3^{\text {rd }}$ day of hospitalization the blood culture showed no growth and urine culture showed the presence of an $A$. baumanii which was sensitive only to imipenam-cilastatin and tigecyclin. Since the patient's general condition and vital signs improved dramatically without any antibiotic, so we observed the patient in the inpatient department for 7 days and then discharged for follow-up in the outpatient department with repeat urine culture after 1 month. The repeated urine culture showed the same organism and the same susceptibility test. The patient was asymptomatic all through out.

\section{CASE REPORT-2}

A 54 year old gentleman who was a known case of Diabetes for last 8 years and was on Metformin $500 \mathrm{mg}$ twice daily presented to the emergency department with fever for 5 days, breathlessness and cough for last 2 days. There was no significant travel or treatment history in recent past. At the time of admission, the patient was febrile- $100.4^{\circ} \mathrm{F}$, pulse $110 / \mathrm{min}, \mathrm{BP}-140 / 90 \mathrm{~mm} \mathrm{Hg}$, the respiratory rate was $36 / \mathrm{min}$. On examination of chest trachea was central, impaired note on percussion in right mid clavicular line from the $4^{\text {th }}$ intercostal space and below and below, the $6^{\text {th }}$ intercostal space and below in mid axillary line and the $8^{\text {th }}$ intercostal space and below in mid scapular line. There was tubular bronchial breath sound and occasional coarse crackles on the areas of dull note. Vocal fremitus and vocal resonance was increased over the corresponding areas. CVS, GI system and CNS examination did not reveal any significant abnormality. The patient was admitted with a provisional diagnosis of right middle lobe community acquired pneumonia with differential diagnosis of lung malignancy and tuberculosis. Routine hematological and biochemical parameters were within normal limits. Xray chest showed right middle lobe consolidation (Figure 1). Sputum was sent for Gram staining and AFB stain. Blood, urine and sputum were sent for aerobic routine cultures. The patient was shifted to high dependent unit and was put on S/C insulin, I/V Amoxy-Clav, I/V Theophylline. The patient became hypotensive and the condition worsened after six hours and the patient was shifted to ICU. Mechanical ventilation was started with Norepinephrine in titrated doses and the antibiotic was changed over to IV Piperacillin-Tazobactum, IV Amikacin and IV Metronidazole. On the $2^{\text {nd }}$ day, the patient also remained hypotensive, sensorium deteriorated and the TLC was 26,000 with DLC showing N-76\% and the sputum showed short Gram negative rods without any acid fast bacilli. Hydrocortisone $50 \mathrm{mg}$ I/V 8 hourly was added to the regimen to combat septic shock. On the third day, the sputum culture showed $A$. baumanii which was sensitive only to imipenam-cilastatin, tigecyclin and polymyxin B. Unfortunately the patient succumbed 2 hours after starting I/V Imipenam $1 \mathrm{Gm}$.

\section{DISCUSSION}

Acinetobacter spp, has emerged as one of the common nosocomial pathogens. A variety of human infections caused by Acinetobacter spp, has been described such as pneumonia (endotrachial tube or tracheotomy induced), skin and wound infections, urinary tract infection (after instrumentation), meningitis (in neurosurgery patients), peritonitis (peritoneal dialysis induced). Sporadic cases of endocarditis, conjunctivitis, osteomylitis and synovitis have also been described. Being ubiquitous in the environment strains of Acinetobacter are widely distributed in hospital environment. They easily colonize the oral cavity, airways, urinary and GI tract. They are also found on moist skin areas of both patients and health care workers.

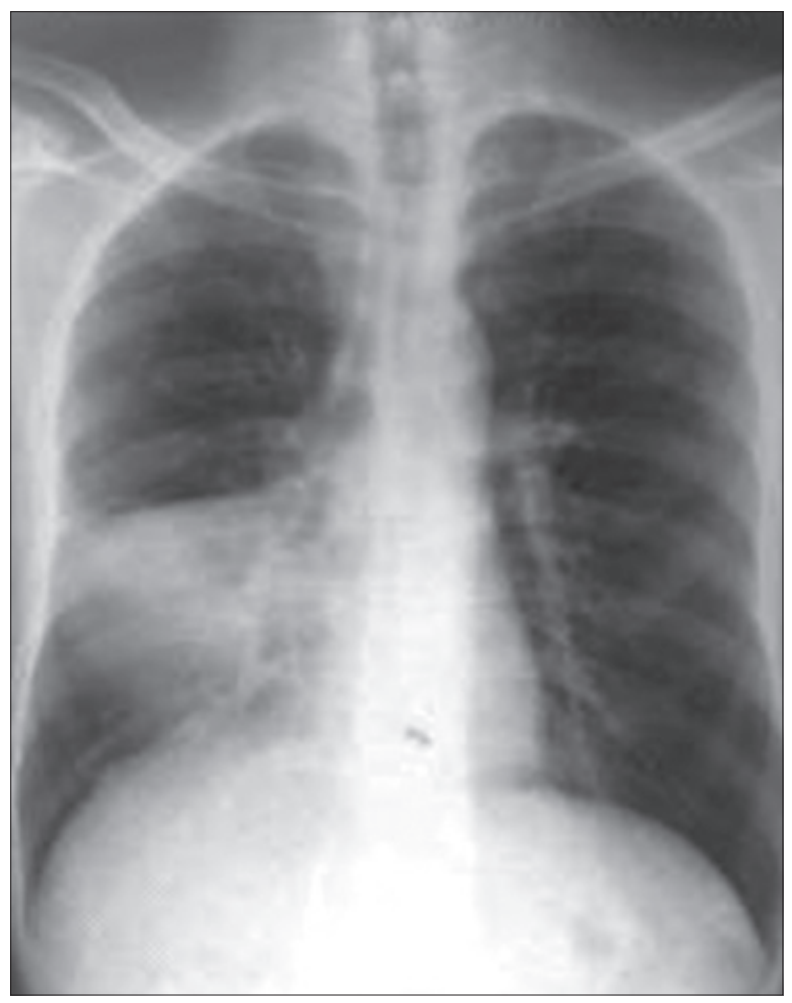

Figure 1: Right middle lobe pneumonia 
Acinetobacter can be a pathogen or comenssal in the hospital environment. When isolated from blood, they are invariably considered to be pathogens but when isolated from other samples like that of wound surfaces and urine they can be either comenssal or pathogen.

In the first case report, we documented a case of asymptomatic MDR A. baumanii infection which probably colonized the urinary tract during indwelling catheterization in hospital and persisted even after 50 days of discharge from the hospital. Though many cases of Acinetobacter colonization of urinary tract are documented during inhospital stay of the patients, we could not find any other article regarding the persistence of Acinetobacterin urinary tract even after discharge from the hospital. SP Yavankar et al have described asymptomatic MDR Acinetobacter on the moist skin of tribal population of Maharastra but did not mention any information on the urinary tract. In this case we did not treat the infection as we were lucky enough that the patient was asymptomatic all throughout. If the patient would have been symptomatic because of some other reason, we might have tempted to treat with Imipenam or Tigecyclin thinking it to be Acinetobacter urosepsis.

In the second case, we dealt with a case of community acquired pneumonia and the pathogen was $A$. baumanii. Probably the patient could have been saved with timely management of the patient usuing the appropriate antibiotic. $A$. baumanii as a cause of community acquired pneumonia is very rare in India. ${ }^{5}$ Only one case of community acquired pneumonia has been documented in India from the valley of Kashmir. ${ }^{6}$ Both of our cases were immune-compromised (diabetic).

\section{CONCLUSION}

A suspicious clinician and a very good microbiology laboratory is essential for the diagnosis of $A$. baumanii infections. Though $A$. baumanii infections are most commonly nosocomial, but they can also be community acquired as in our second case. They must be treated timely pending susceptibility test with carbapenams if the symptoms are not improving. As there is no clear cut guideline regarding the treatment of $A$. baumanii infections, it is best left to the clinicians to be judicious and apply his best scientific knowledge in an artistic sense.

\section{REFERENCES}

1. Washington C. Winn Jr (2006). Koneman's colour atlas and textbook of diagnostic microbiology. $6^{\text {th }}$ edition. 2012: 353-355p.

2. Yavankar SP, Pardesi KR and Chopade BA. Species distribution and physiological characterization of Acinetobacter genospecies from healthy human skin of tribal population in India. Indian $\mathrm{J}$ Med Microbiol 2007; 25: 336-345.

3. Scott P, Deye G, Srinivasan A, Murray C, Moran K, Ed Hulten, et al: An outbreak of multidrug-resistant Acinetobcter baumaniicalcoaceticus complex infection in US military health care system associated with military operation in Iraq. Clin Infect Dis 44:1577, 2007.

4. Paterson DL. Resistance in gram negative bacteria: Enterobacteriaceae. Am J Infect Control 2006; 34: S20-S28; S64-S73.

5. Gupta D, Agarwal R, Aggarwal AN, Singh N, Mishra N, Khilnani $G C$, et al. Guidelines for diagnosis and management of community-and hospital-acquired pneumonia in adults: Joint ICS/NCCP(I)recommendations. Lung India 2012; 29:27-62.

6. Shah BA, Singh G, Naik MA and Dhobi GN. Bacteriological and clinical profile of Community acquired pneumonia in hospitalized patients. Lung India 2010; 27:54-57. 